\title{
No-reference Image Quality Assessment for Photographic Images Based on Robust Statistics
}

\author{
Zhengda Zeng ${ }^{\mathrm{a}}$, Wenming Yang ${ }^{\mathrm{a}, *}$, Wen Sun $^{\mathrm{a}}$, Jing-Hao Xue ${ }^{\mathrm{b}}$, Qingmin Liao $^{\mathrm{a}}$ \\ ${ }^{a}$ Shenzhen Key Lab. of Info. SciछTech/Shenzhen Engineering Lab. of IS\&SCP, \\ Department of Electronic Engineering/Graduate School at Shenzhen, Tsinghua University, \\ China \\ ${ }^{b}$ Department of Statistical Science, University College London, UK
}

\begin{abstract}
No-reference image quality assessment (NR-IQA) is developing rapidly, but there lacks of research on exploring robust statistics to improve the prediction accuracy and monotonicity of NR-IQA algorithms, in particular for assessing photographic images captured by different digital cameras where a variety of unknown distortions may happen. Hence this paper proposes a novel robuststatistics-based NR-IQA method (termed RSN) for photographic images. In RSN, we present three types of features based on robust statistics: robust natural scene statistics of multiple components, robust multi-order derivatives, and robust complementary features in the frequency domain. Then support vector regression is applied to predict image quality using the extracted features. Experimental results show that RSN remarkably outperforms state-of-the-art NR-IQA methods on the CID2013 database of photographic images, as well as on the popular LIVE and TID2013 databases.

Keywords: Image quality assessment (IQA), no-reference/blind IQA, camera image, robust statistics, natural scene statistics (NSS).

\footnotetext{
* Corresponding author.

Email addresses: zzhengda92@gmail.com (Zhengda Zeng), yangelwm@163.com liaoqm@sz.tsinghua.edu.cn (Qingmin Liao)
} (Wenming Yang), 1213755392@qq.com (Wen Sun), jinghao.xue@ucl.ac.uk (Jing-Hao Xue),
\end{abstract}




\section{Introduction}

With the rapid popularity of smart phones, images produced by digital cameras are becoming an increasingly important medium of access to information. However, they are still prone to be distorted during acquisition, compression, 5 transmission, processing and restoration. Distortions impair the image quality and even the understanding of the information contained. Therefore, it is timely to automatically predict the perceptual quality of photographic images shown in 1 .

Image quality assessment (IQA) is performed in two ways: subjective and objective. Subjective IQA is accurate to reflect human's visual perception, but it is time-consuming, cumbersome and often impractical. Hence, it is necessary to develop objective IQA algorithms, which can evaluate image quality automatically and accurately. According to the information availability of pristine reference images, objective IQA methods can be divided into three cate-

15 gories: full-reference (FR), reduced-reference (RR) and no-reference (NR) [1] [] . When reference images are available, the FR-IQA methods, such as SSIM [1, GMSD [7, VSI 8], LLM [9, MvSSIM [10] and PQIC [11, can be applied to directly evaluate the difference between a distorted image and its reference image. For RR-IQA, part of the reference image information is available to calculate the quality scores. In reality, the reference images are often unavailable, hence FR-IQA and RR-IQA are infeasible and NR-IQA should be used instead. The state-of-the-art NR-IQA methods include DIIVINE [12, BLIINDS-II [13, BRISQUE [14, CNN [15], HOSA [16], IDEAL [17], method in [18, etc. This paper concentrates on NR-IQA for photographic images.

The aforementioned NR-IQA methods, unfortunately, have been validated only on images affected by artificial distortions, such as those in the LIVE [19] and TID2013 20] databases. Photographic images often mix a variety of unpredictable distortions for which a single type of features, e.g. sharpness, illumination and colour information, can predict poorly. Hence, some algorithms 
hybrid approach is illustrated to utilise the natural scene statistics (NSS) modelling and the quality-aware interpretable features, such as sharpness and noise. The method in 22 combines sharpness measure and compound noise estimation. The BQIC method [23] addresses this problem from two aspects, NSS and 35 local sharpness.
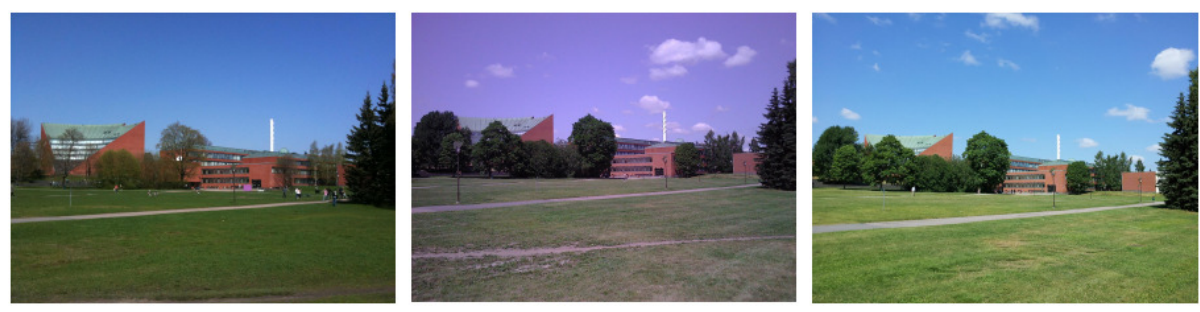

Figure 1: Examples of photographic images in the CID2013 database. The quality scores (MOS) of the images are 31.3, 47.8 and 87.9 in order.

Despite these promising methods, there is still a lack of research on exploring robust statistics to improve the prediction accuracy and monotonicity of IQA (including NR-IQA) algorithms for photographic images . Robust statistics 24], a valid discipline of statistical to make popular statistics less affected by outliers and perform better over a wider range of probability distributions, has been widely applied in digital image processing, such as image thresholding [25], image denoising [26], image registration [27] and face recognition [28]. Therefore in this paper, we proposes a novel Robust Statistics-based NR-IQA method (termed RSN) for photographic images. In RSN, we propose three types of new robust statistical features: robust natural scene statistics of multiple components, robust multi-order derivatives, and robust complementary features in the frequency domain.

More specifically, these three types of new robust features are extracted as follows. Firstly, we construct five components of an image (luminance compo50 nent, two chrominance components, maximum local variation component and minimum local variation component [29]), and compute the median subtracted contrast normalised coefficients (MDSCN), as the robust counterpart of the 
mean subtracted contrast normalised coefficient (MSCN) [14, 30], using some robust summary statistics. From the MDSCN coefficients, the variance, shape, skewness, kurtosis and entropy are extracted as robust NSS features. Secondly, we propose the robust multi-order derivative features, which are composed of the medians and mean absolute deviations (MADs) of the colour image and its first three derivatives. Thirdly, we utilise some complementary features in the frequency domain, which include the robust saliency features that we derive from the statistical property of saliency maps and the sharpness measured by the FISH characteristic [31. When a feature vector is constituted by the three types of features, support vector regression (SVR) is used to map the feature vector to image quality.

Besides our above-mentioned contributions to feature extraction, we shall 65 use experiments to demonstrate that the proposed robust features and RSN method can achieve remarkable improvement in prediction monotonicity and accuracy on the CID2013 database of photographic images, as well as on the popular LIVE and TID2013 databases.

The rest of the paper is organized as follows. Section 2 presents the details of the proposed camera image quality assessment algorithm. In Section 3 the experimental details and results are presented, and the effectiveness of our algorithm is validated. Finally, some conclusions and future work are presented in Section 4

\section{The proposed method}

The framework of our new RSN method is shown in Fig 2, mainly including two parts: feature extraction and regression-model learning. In [21 23, when various features are fused, the IQA performance is largely improved, especially for photographic images affected by multiple unknown distortions. In this paper we shall show that using our proposed robust NSS features, robust multi-order derivative features and robust complementary features can offer even much further improvement. 


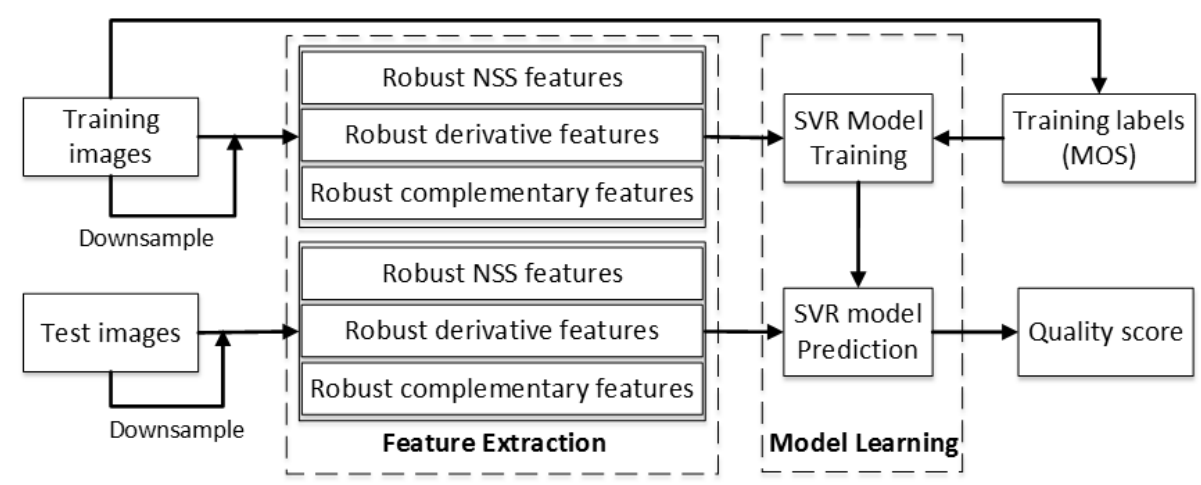

Figure 2: The framework of the proposed robust statistics-based no-reference IQA method for photographic images (RSN).

\subsection{Robust NSS features}

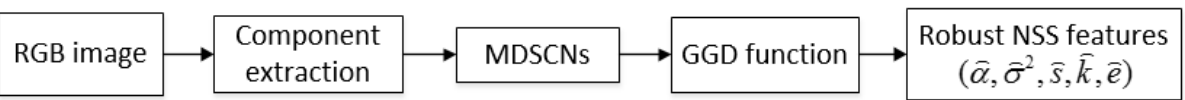

Figure 3: The process of extracting robust NSS features.

In this subsection, motivation and implementation of our robust NSS features are provided. There is a well-known hypothesis about NSS 14, 32 that natural images possess certain statistical properties that can be modified by the presence of distortions. The NSS approach has been successfully applied in NR-IQA through several image domains, such as the wavelet domain in DIIVINE [12, the DCT domain in BLIINDS-II [13] and the spatial domain in BRISQUE [14] and IDEAL [17. Our robust NSS features are based on the NSS in the spatial domain because of the pursuit of lower computational complexity and better performance. Recently, the characteristics of chrominance components have been gradually exploited to complement the features in luminance components. The IDEAL [17] proposes four statistic properties from colour representations: hue, saturation, opponent angle and spherical angle. Hence, we attempt to exploit the statistical properties of luminance components $I_{L}$ along with two different chrominance components $\left(I_{M}, I_{N}\right)$ in the LMN colour 
space 33. Also, in MLV 29], the distribution of maximum local variation component $I_{\text {MaxL }}$ can be parameterised with the generalised Gaussian distribution (GGD), which is easy to extend to minimum local variation component $I_{\text {MinL }}$. That is, we extract the robust NSS features on multiple components, the diagram of which is shown in Fig 3 .

Specifically, given an RGB image of size $H \times W$, we first convert it into the LMN colour space 33 , obtaining a luminance component $I_{L}$ and two chrominance components $I_{M}$ and $I_{N}$.

$$
\left[\begin{array}{c}
I_{L} \\
I_{M} \\
I_{N}
\end{array}\right]=\left[\begin{array}{ccc}
0.06 & 0.63 & 0.27 \\
0.30 & 0.04 & -0.35 \\
0.34 & -0.60 & 0.17
\end{array}\right]\left[\begin{array}{c}
I_{R} \\
I_{G} \\
I_{B}
\end{array}\right] .
$$

Then, inspired by the statistical properties of MLV [29] to describe sharpness, we propose to augment the feature space by adding two new order-statisticsbased components: the maximum local component $\left(I_{M a x L}\right)$ and minimum local component $\left(I_{\text {MinL }}\right)$ of the luminance component $I_{L}$ :

$$
I_{\text {Max } L}=\left(\begin{array}{ccc}
\psi\left(I_{L}(1,1)\right) & \ldots & \psi\left(I_{L}(1, W)\right) \\
\vdots & \ddots & \vdots \\
\psi\left(I_{L}(H, 1)\right) & \ldots & \psi\left(I_{L}(H, W)\right)
\end{array}\right)
$$

where each entry $\psi\left(I_{L}(x, y)\right)$ of pixel $I(x, y)$ is defined as

$$
\psi\left(I_{L}(x, y)\right)=\max _{(i, j) \in N_{(x, y)}}\{|I(i, j)-I(x, y)|\},
$$

110 component $I_{\text {MinL }}$ can be similarly obtained.

Then, for each of the five components $I_{k}$, we propose the median subtracted contrast normalised (MDSCN) coefficient $\hat{I}_{k}$ as the new robust counterpart of MSCN:

$$
\hat{I}_{k}(x, y)=\frac{I_{k}(x, y)-\operatorname{med}_{k}(x, y)}{\operatorname{RMD}_{k}(x, y)+C}
$$

115

where index $k \in\{L, M, N, \operatorname{Max} L, \operatorname{MinL}\}$ denotes a component; $C$ is a small constant preventing the denominator from tending to zero; $\operatorname{med}_{k}(x, y)$ is the 
local median, a robust order-statistic for data location; and $\operatorname{RMD}_{k}(x, y)$ is the local root median square error, a statistic for data variability:

$$
\operatorname{RMD}_{k}(x, y)=\sqrt{\frac{1}{\left|N_{(x, y)}\right|} \sum_{(i, j) \in N_{(x, y)}}\left(I_{k}(i, j)-\operatorname{med}_{k}(x, y)\right)^{2}},
$$

where $\left|N_{(x, y)}\right|$ denotes the size of the local image patch $3 \times 3$.

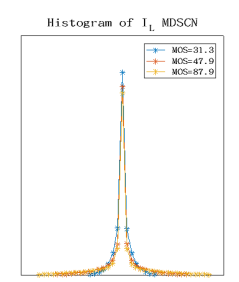

Histogram of $\mathrm{I}_{1}$ MSCN

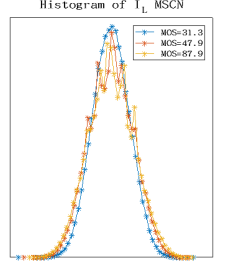

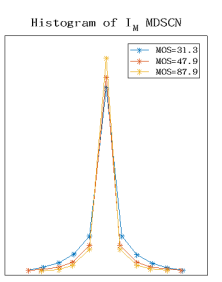
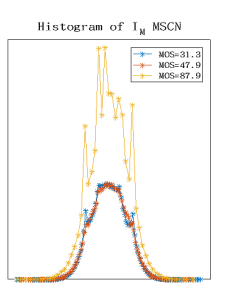
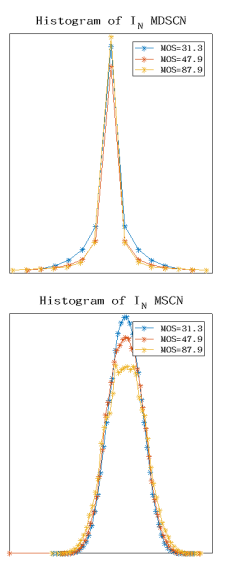
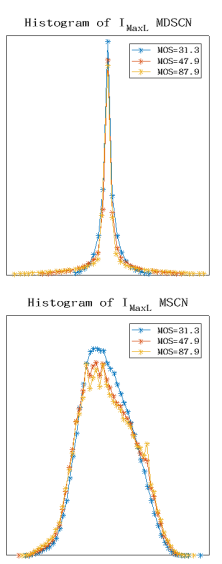
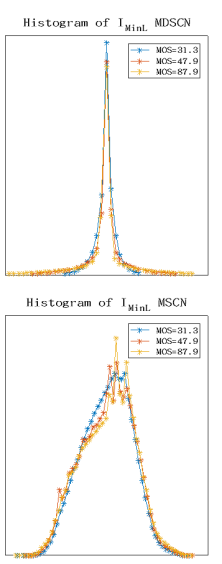

Figure 4: Histograms of the MDSCN and MSCN coefficients for five components of the three photographic images shown in Fig. 1

We analyze histograms of the robust MDSCN and non-robust MSCN coefficients for different components. Three photographic images captured in the same scene are shown in Fig. 1] Their subjective quality scores (MOS) are 31.3, 47.8 and 87.9 in order, in which the higher the subjective score, the better the quality. The histograms of the MDSCN and MSCN coefficients for five components $\left\{\hat{I}_{L}, \hat{I}_{M}, \hat{I}_{N}, \hat{I}_{M a x L}, \hat{I}_{M i n L}\right\}$ of the three photographic images are shown in Fig. 4. The first row corresponds to MDSCN coefficients (robust version) and the second row corresponds to MSCN coefficients (non-robust version). The blue, red and yellow dotted lines in each histogram correspond to the three images in Fig. 1] respectively. It can be seen that the histograms of MSCN coefficients not only contains many outliers, but also may tilt to one side, such as the distributions for Lmax and Lmin components. However, these two problems can be overcome in the robust MDSCN coefficients as illustrated in Fig 4 
In addition, the histograms of robust MDSCN coefficients for photographic images with different quality scores are more recognizable than the corresponding non-robust version.

It was shown that the MSCN coefficients of a distorted grey-image can be effectively characterised by GGDs [14]. We also use GGDs to model the distributions of the MDSCN coefficients. Therefore, we finally use the parameters of GGDs to summarise the statistical properties of these new coefficients. Let us take the luminance component as example to illustrate our process of feature extraction.

For an MDSCN coefficient $\hat{I}_{L}(x, y)=z_{\hat{I}_{L}}$, its probability density function of the GGD with zero mean is

$$
f\left(z_{\hat{I}_{L}} ; \alpha_{\hat{I}_{L}}, \beta_{\hat{I}_{L}}\right)=\frac{\alpha_{\hat{I}_{L}}}{2 \beta_{\hat{I}_{L}} \Gamma\left(\frac{1}{\alpha_{\hat{I}_{L}}}\right)} \exp \left\{-\left(\frac{\left|z_{\hat{I}_{L}}\right|}{\beta_{\hat{I}_{L}}}\right)^{\alpha_{\hat{I}_{L}}}\right\},
$$

where shape parameter $\alpha_{\hat{I}_{L}}$ and scale parameter $\beta_{\hat{I}_{L}}$ satisfy $\beta_{\hat{I}_{L}}=\sigma_{\hat{I}_{L}} \sqrt{\frac{\Gamma\left(1 / \alpha_{\hat{L}_{L}}\right)}{\Gamma\left(3 / \alpha_{\hat{I}_{L}}\right)}}$. Hence, two parameters $\left(\alpha_{\hat{I}_{L}}, \sigma_{\hat{I}_{L}}^{2}\right)$ for shape and variance are sufficient to represent the GGD of $\hat{I}_{L}$; they are estimated as with [34] over each image scale.

Besides these two estimated parameters, we also choose three other sample statistics to make a more comprehensive set of robust NSS features from the MDSCN coefficients: $s_{\hat{I}_{L}}$, the sample skewness; $k_{\hat{I}_{L}}$, the sample kurtosis; and $e_{\hat{I}_{L}}$, the entropy. As with 35, they are calculated as

$$
\begin{gathered}
d\left(\hat{I}_{L}\right)=\sqrt{\mu\left(\hat{I}_{L}-\mu\left(\hat{I}_{L}\right)\right)^{2}}, \\
s_{\hat{I}_{L}}=\frac{\mu\left[\left(\hat{I}_{L}-\mu\left(\hat{I}_{L}\right)\right)^{3}\right]}{d\left(\hat{I}_{L}\right)^{3}}, \\
k_{\hat{I}_{L}}=\frac{\mu\left[\left(\hat{I}_{L}-\mu\left(\hat{I}_{L}\right)\right)^{4}\right]}{d\left(\hat{I}_{L}\right)^{4}}-3
\end{gathered}
$$

and

$$
e_{\hat{I}_{L}}=-\sum_{j} p_{j}\left(\hat{I}_{L}\right) \log _{2}\left(p_{j}\left(\hat{I}_{L}\right)\right)
$$

where $\mu(\cdot)$ is the sample mean and $p(\cdot)$ is the histogram. 
Hence, five sample estimators of $\left(\alpha, \sigma^{2}, s, k, e\right)$ for each of the five MDSCN components $\left\{\hat{I}_{L}, \hat{I}_{M}, \hat{I}_{N}, \hat{I}_{M a x L}, \hat{I}_{M i n L}\right\}$ are chosen as the robust NSS features.

\subsection{Robust multi-order derivative features}
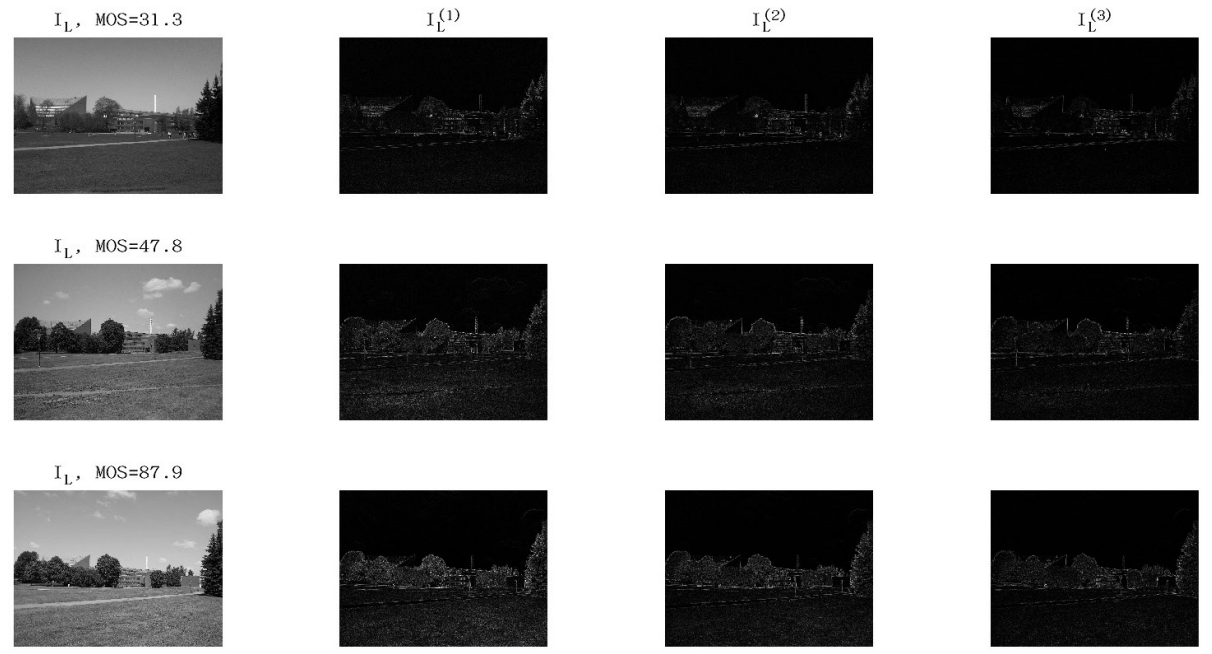

Figure 5: Examples of multi-order derivatives. Different rows correspond to photographic images with different quality scores $(31.3,47.8,87.9)$ shown in Fig. 1 and different columns correspond to different order derivatives $\left(I_{L}^{(0)}, I_{L}^{(1)}, I_{L}^{(2)}, I_{L}^{(3)}\right)$.

The multi-order derivatives of luminance components (gray images) are shown in Fig. 5 . Different rows correspond to photographic images with different quality scores $(31.3,47.8,87.9)$ shown in Fig. 1 and different columns correspond to the luminance component and its first three order derivatives $\left(I_{L}^{(1)}, I_{L}^{(2)}, I_{L}^{(3)}\right)$. We treat $I_{L}$ as its own zero-order derivative $I_{L}^{(0)}$. According to [36], the first order derivative $I_{L}^{(1)}$ is usually related to the slope and elasticity of image surface, the second order derivative $I_{L}^{(2)}$ is used to capture the curvature, and higher order derivatives can capture detailed discriminative information 37. For photographic images with different quality scores in the same scene, the characteristics of the same derivative, such as structure, edge, texture are different. Thus, higher order derivatives can capture the details of image structure in the spatial domain, and have been successfully applied to 
filter of size $3 \times 3$ to calculate the derivatives for its simplicity.

It is well known that, when the histogram is skew or heavy-tailed or when there are outliers, the median and mean absolute deviation (MAD) are more robust estimators of location and variation than the mean and standard deviation, respectively [25, 38]. Hence we propose to use the medians and MADs of the colour image $\left(I_{L}, I_{M}, I_{N}\right)$ and the first three derivatives $\left(I_{L}^{(1)}, I_{L}^{(2)}, I_{L}^{(3)}\right)$ of the luminance component $I_{L}$ as our robust multi-order derivative features.

Given a grey-image $I_{L}$ (or say $I_{L}^{(0)}$ ), the $n$th order derivative image $I_{L}^{(n)}$ is calculated from the $(n-1)$ th order one $I_{L}^{(n-1)}$ :

$$
I_{L}^{(n)}=\sqrt{\left(I_{L}^{(n-1)} * h_{x}\right)^{2}+\left(I_{L}^{(n-1)} * h_{y}\right)^{2}},
$$

where $h_{x}$ and $h_{y}$ are the Prewitt filters along the horizontal and vertical directions, respectively.

Then we choose the medians and MADs of the $\left\{I_{L}, I_{M}, I_{N}, I_{L}^{(1)}, I_{L}^{(2)}, I_{L}^{(3)}\right\}$ as the robust multi-order derivative features; MAD is defined as

$$
\operatorname{MAD}_{k}^{(n)}=\frac{1}{\left|I_{k}^{(n)}\right|} \sum_{(i, j) \in I_{k}^{(n)}}\left|I_{k}^{(n)}(i, j)-\operatorname{med}_{k}^{(n)}\right|
$$

where index $k \in\{L, M, N\}$ denotes a component, index $n \in\{0,1,2,3\}$ denotes the derivative order, and $\left|I_{k}^{(n)}\right|$ denotes the size of the component $I_{k}^{(n)}$.

\subsection{Robust complementary features}

The above-defined robust NSS and derivative features are extracted from the spatial domain. To make the feature space more complete, we propose to extract the saliency features and sharpness from the frequency domain as a complement to the robust spatial features.

Firstly, the saliency features have been successfully applied in the FR-IQA algorithm VSI [8]. Considering the assumption that an image's visual saliency map has a close relationship with its perceptual quality since both of them depend on how the human visual system perceives an image [39], we also integrate 


\section{Experimental results}

\subsection{Experimental settings}

We use the newly released CID2013 [20] database of photographic images to evaluate our method and compare it with existing state-of-the-art NR-IQA 
for multi-degraded images without references. It consists of 474 photographic images obtained by 79 different types of cameras in 8 different shooting scenes. Moreover, in order to validate the versatility of our method, experiments on two popular databases, the LIVE database [19] and the TID2013 database [42], JPEG2000, JPEG, Gaussian blur, white noise and fast fading, of 29 original images. The TID2013 database is constructed from 25 reference images and includes 3000 images with 24 types of distortion at five distortion levels.

Considering the non-linear relationship between the predicted score and the subjective score, logistic functions are usually applied to convert the predicted scores into the same scale as the subjective scores [19, 20, 43]. In this paper, we adopt the five-parameter logistic fit function used in [20]:

$$
f(\hat{x})=\beta_{1}\left\{\frac{1}{2}-\frac{1}{1+\exp \left(\beta_{2}\left(\hat{x}-\beta_{3}\right)\right)}\right\}+\beta_{4} \hat{x}+\beta_{5},
$$

where $\hat{x}$ is the predicted score, and $\beta_{1}$ to $\beta_{5}$ are the function parameters to be determined by minimising the MSE between $f(\hat{x})$ and the true subjective score.

In order to evaluate the performance of algorithms, three common evaluation criteria are used: Spearman's rank ordered correlation coefficient (SROCC), Pearson's linear correlation coefficient (PLCC) and the root mean square error (RMSE). Among them, SROCC is to assess prediction monotonicity, while PLCC and RMSE are to assess prediction accuracy.

\subsection{Comparison on the CID2013 database}

We compare our method with some state-of-the-art NR-IQA methods including the present best method on the CID2013 database. These methods are: BRISQUE [14, BLIINDS-II [13, IDEAL [17], RISE [44, the method in [21, CIQM [45, BQIC [23, 46] and the method in 22]. The experimental setting 245 with leave-one-out cross validation [47] is commonly used in many NR-IQA method [18, 21, 35]. Since the CID2013 database is divided into six sets based on different image scenes, we adopted six-fold leave-one-out cross validation [21]: 
Table 1: Performance on the CID2013 database. NSRN: the non-robust counterpart of RSN.

\begin{tabular}{c|r|r|r}
\hline & PLCC & SROCC & RMSE \\
\hline BRISQUE [14] & 0.781 & 0.784 & 14.140 \\
BLIINDS-II [13] & 0.639 & 0.635 & 17.409 \\
RISE [44] & 0.793 & 0.769 & 13.782 \\
Method in [21] & 0.799 & 0.815 & \\
Method in [22] & 0.818 & 0.793 & 12.948 \\
CIQM [4] & 0.817 & 0.805 & 12.887 \\
BQIC [23] & 0.829 & 0.821 & 12.680 \\
IDEAL [17] & 0.814 & 0.817 & 13.302 \\
NRSN & 0.834 & 0.823 & 12.043 \\
RSN & $\mathbf{0 . 9 0 9}$ & $\mathbf{0 . 8 9 0}$ & $\mathbf{9 . 3 4 1}$ \\
\hline
\end{tabular}

we divided the image database into six sets based on image scenes, and used five image sets for training the SVR model and the remaining one for testing. The procedure repeats six times, each time with a different test set, and the mean results of the six-fold evaluation of SROCC, PLCC and RMSE are taken.

The experimental results are listed in Table1. Our method performs remarkably better than other methods in terms of all three measures, PLCC, SROCC and RMSE, as well as its non-robust counterpart (NRSN). Even compared with 255 the latest method BQIC [23], our PLCC and SROCC are more than 9\% and $8 \%$ better, respectively. This indicates a better correlation of our method with the human visual perception of the distorted images than other state-of-the-art NR-IQA methods.

It is necessary to further analyse the effectiveness of each type of robust features and the different combinations of them. The experimental results are listed in Table 2. It shows that the robust NSS features (NSS*), that use the robust MDSCN to normalise components, are superior to the NSS features that use the ordinary MSCN. Similarly, the robust multi-order derivative features (Derivatives*), that extract the medians and MADs as features, achieve bet- 
Table 2: Analysis of each type of features on the CID2013 database. NSS*: robust NSS features; Derivatives*: robust multi-order derivative features; Complement*: robust complementary features (saliency and sharpness).

\begin{tabular}{c|r|r|r}
\hline features used & PLCC & SROCC & RMSE \\
\hline NSS & 0.718 & 0.712 & 15.402 \\
NSS* & 0.875 & 0.866 & 10.655 \\
Derivatives & 0.829 & 0.812 & 12.339 \\
Derivatives* & 0.860 & 0.854 & 10.947 \\
Complement* & 0.822 & 0.803 & 12.511 \\
\hline NSS*+Derivatives* $^{*}$ & 0.885 & 0.877 & 10.128 \\
NSS*+Complement* $^{*}$ & 0.877 & 0.870 & 10.454 \\
Derivatives*+Complement* & 0.873 & 0.864 & 10.753 \\
\hline NRSN & 0.834 & 0.823 & 12.043 \\
RSN & $\mathbf{0 . 9 0 9}$ & $\mathbf{0 . 8 9 0}$ & $\mathbf{9 . 3 4 1}$ \\
\hline
\end{tabular}

ter performance than their ordinary counterparts (Derivatives) that use the mean and standard variation. Furthermore, the performance of combined features (NSS* and Derivatives*) is better than that of separate features (NSS* or Derivatives*). Similar patterns are with other combined features. In the end, when all three types of features are used together, we can obtain the best performance. This indicates that these three types of robust features are complementary to some extent and they are superior to their non-robust counterparts.

Moreover, in order to demonstrate the collective added value of the robust features, we also implement a non-robust version of our RSN, by replacing the involved robust statistics with their ordinary counterparts. We call this nonrobust version 'NRSN'. As shown in Table 1 (or Table 2), NRSN performs worse than RSN, its robust version.

\subsection{Comparison on the LIVE and TID2013 databases}

To verify the versatility of our approach, we also compare it with some stateof-the-art NR-IQA approaches on the popular LIVE and TID2013 databases. 
Table 3: Performance on the LIVE and TID2013 databases.

\begin{tabular}{c|r|r|r|r}
\hline & \multicolumn{2}{|c|}{ LIVE } & \multicolumn{2}{c}{ TID2013 } \\
\cline { 2 - 5 } & PLCC & SROCC & PLCC & SROCC \\
\hline BRISQUE [14] & 0.939 & 0.940 & 0.800 & 0.719 \\
BLIINDS-II [13] & 0.930 & 0.931 & 0.736 & 0.644 \\
DIIVINE [12] & 0.917 & 0.916 & 0.641 & 0.558 \\
IDEAL [17] & 0.946 & 0.941 & 0.767 & 0.719 \\
UNIQUE [48] & 0.956 & 0.952 & 0.868 & 0.860 \\
NRSN & 0.974 & 0.971 & 0.896 & 0.884 \\
RSN & $\mathbf{0 . 9 7 5}$ & $\mathbf{0 . 9 7 6}$ & $\mathbf{0 . 9 0 6}$ & $\mathbf{0 . 9 0 6}$ \\
\hline
\end{tabular}

The approaches chosen include the mainstream BLIINDS-II [13, DIIVINE [12, and BRISQUE [14, as well as the recently published IDEAL [17] and UNIQUE [48] methods. PLCC and SROCC are adopted as evaluation measures, and the experimental settings remain unchanged.

The experimental results in Table 3 show that the proposed method (RSN) is the best in terms of both SROCC and PLCC on the LIVE and TID2013 databases; that is, it is superior to the compared NR-IQA methods and its non-robust version (NRSN) in prediction monotonicity and accuracy.

In summary, our robust statistics-based RSN method performs strongly for both the complex mixed-distortion photographic images in the CID2013 database and the simulated-distortion images in the LIVE and TID2013 databases.

\subsection{Computational complexity}

We now demonstrate that our method does not expend substantial computation. We compare the overall computational complexity of our RSN with other mainstream NR-IQA methods on the CID2013 database, such as DIIVINE [12, BLIINDS-II [13, BRIQUE [14], IDEAL [17, and Method in 22]. In Table 4 . we list the time taken (in seconds) to calculate each quality measure on a photographic image of resolution 12001400 on a $2.4 \mathrm{Ghz}$ Inter Xeon CPU with 64 GB RAM system running Xubuntu 16.04 OS. We use non-optimized MAT- 
LAB codes for all of these algorithms to ensure a fair comparison. It can be observed that the computational time of our method is ranked under the middle among the compared methods although it is higher than its non-robust version (NRSN).

Table 4: Complexity analysis. NSRN: the non-robust counterpart of RSN.

\begin{tabular}{c|r}
\hline Algorithm & Time(seconds) \\
\hline DIIVINE [12] & 85.836 \\
BLIINDS-II [13] & 400.178 \\
BRIQUE [14] & 1.027 \\
IDEAL [1] & 5.336 \\
Method in [22] & 7.150 \\
NRSN & 1.908 \\
RSN & $\mathbf{4 . 4 8 5}$ \\
\hline
\end{tabular}

The computational time of different stages of our algorithm is shown in Table 5. We can observe that the time consumption of prediction with the SVR model is almost negligible, and most computational time is consumed in feature extraction. The extractions of three robust features take up different proportions of computational time, of which the extraction of robust NSS feature accounts for the largest proportion.

Table 5: Percentage of computational time on individual stages. NSS*: robust NSS features; Derivatives*: robust multi-order derivative features; Complement*: robust complementary features (saliency and sharpness).

\begin{tabular}{c|r}
\hline Processing stage & Percentage of Delay \\
\hline NSS* $^{*}$ & $77.40 \%$ \\
Derivatives* & $8.70 \%$ \\
Complement* & $13.85 \%$ \\
\hline SVR model & $0.05 \%$ \\
\hline
\end{tabular}




\subsection{Discussion}

310 significantly superior to other NR-IQA algorithms on the CID2013 database.

Firstly, the non-robust version NRSN with the NSS feature, the derivative feature and the complementary feature in the frequency domain lays the foundation. The performance of the NRSN algorithm is slightly better than other algorithms as shown in Table 1.

Secondly, the strategy based on robust statistics greatly improves the performance of non-robust features. Photographic images are impaired with a variety of unknown distortions, which causes that distributions of photographic images are scattered and there are many outliers in the distributions. Thus, the robust statistics are proposed to replace general statistics, which significantly improves the non-robust version (NRSN). Specifically, the MDSCN coefficients are used to replace the MSCN coefficients in the extraction of the NSS feature and the complementary feature. The median and MAD are used to replace the mean and variance in the extraction of derivative feature, respectively. The ex325 periment shown in Table 2 has demonstrated that any single robust statistical feature is significantly superior to its non-robust statistical feature, as well as the algorithm (RSN) based on robust statistics is significantly superior to its corresponding non-robust version (NRSN).

Finally, in many cases, a single feature is not enough to characterize the quality of photographic images with multiple unknown distortions. Thus, we integrate three features based on robust statistics (robust NSS feature, robust derivative feature and robust complementary feature) in our algorithm. The effectiveness of fusion strategy is proved good in the above section.

\section{Conclusion}

In this paper, we proposed a novel NR-IQA method termed RSN for photographic images based on robust statistics. Three new types of robust features 
are constructed: 1) robust NSS features on multiple components by normalising each component to median subtracted contrast normalised (MDSCN) coefficients and incorporating the information of skewness, kurtosis and entropy; 2) robust multi-order derivative features by utilising their medians and mean absolute derivations (MADs); and 3) the complementary characteristics in the frequency domain - robust saliency and sharpness. Experimental results demonstrated that the proposed RSN achieved remarkable improvement in prediction monotonicity and accuracy on the CID2013 database of photographic images, and the combination of the three types of features was more effective than any other combination.

Our future work is to investigate how to combine deep learning with NR-IQA for photographic images. CNN has been used in NR-IQA [15], but there remains no deep-learning algorithm for resolving NR-IQA for photographic images.

\section{Acknowledgment}

This work was supported by the Natural Science Foundation of China(No.61471216 and 61771276), the National Key Research and Development Program of China (No.2016YFB0101001 and 2017YFC0112500) and the Special Foundation for the Development of Strategic Emerging Industries of Shenzhen(No.JCYJ20170307153940960 and No.JCYJ20150831192224146).

\section{References}

[1] Z. Wang, A. C. Bovik, H. R. Sheikh, E. P. Simoncelli, Image quality assessment: from error visibility to structural similarity, IEEE Transactions on Image Processing 13 (4) (2004) 600-612.

[2] L. Li, Y. Zhou, W. Lin, J. Wu, X. Zhang, B. Chen, No-reference quality assessment of deblocked images, Neurocomputing 177 (2016) $572-584$.

[3] C. Zhang, J. Pan, S. Chen, T. Wang, D. Sun, No reference image quality assessment using sparse feature representation in two dimensions spatial correlation, Neurocomputing 173, Part 2 (2016) 462 - 470. 
[4] X. Xie, Y. Zhang, J. Wu, G. Shi, W. Dong, Bag-of-words feature representation for blind image quality assessment with local quantized pattern, Neurocomputing 266 (2017) 176 - 187.

[5] W. Zhou, S. Zhang, T. Pan, L. Yu, W. Qiu, Y. Zhou, T. Luo, Blind 3d image quality assessment based on self-similarity of binocular features, Neurocomputing 224 (2017) $128-134$.

[6] Q. Li, W. Lin, Y. Fang, Bsd: Blind image quality assessment based on structural degradation, Neurocomputing 236 (2017) 93 - 103.

[7] W. Xue, L. Zhang, X. Mou, A. C. Bovik, Gradient magnitude similarity deviation: a highly efficient perceptual image quality index, IEEE Transactions on Image Processing 23 (2) (2014) 684-695.

[8] L. Zhang, Y. Shen, H. Li, VSI: A visual saliency-induced index for perceptual image quality assessment, IEEE Transactions on Image Processing 23 (10) (2014) 4270-4281.

[9] H. Wang, J. Fu, W. Lin, S. Hu, C. C. J. Kuo, L. Zuo, Image quality assessment based on local linear information and distortion-specific compensation, IEEE Transactions on Image Processing 26 (2) (2017) 915-926. doi:10.1109/TIP.2016.2639451.

[10] R. Zhu, F. Zhou, J.-H. Xue, Mvssim: A quality assessment index for hyperspectral images, Neurocomputing.

[11] S. Wang, K. Ma, H. Yeganeh, Z. Wang, W. Lin, A patch-structure representation method for quality assessment of contrast changed images, IEEE Signal Processing Letters 22 (12) (2015) 2387-2390.

[12] A. K. Moorthy, A. C. Bovik, Blind image quality assessment: From natural scene statistics to perceptual quality, IEEE Transactions on Image Processing 20 (12) (2011) 3350-3364. 
[13] M. A. Saad, A. C. Bovik, C. Charrier, Blind image quality assessment: A natural scene statistics approach in the DCT domain, IEEE Transactions on Image Processing 21 (8) (2012) 3339-3352.

[14] A. Mittal, A. K. Moorthy, A. C. Bovik, No-reference image quality assessment in the spatial domain, IEEE Transactions on Image Processing 21 (12) (2012) 4695-4708.

[15] L. Kang, P. Ye, Y. Li, D. Doermann, Convolutional neural networks for noreference image quality assessment, in: Proceedings of the IEEE Conference on Computer Vision and Pattern Recognition, 2014, pp. 1733-1740.

${ }_{400}$ [16] J. Xu, P. Ye, Q. Li, H. Du, Y. Liu, D. Doermann, Blind image quality assessment based on high order statistics aggregation, IEEE Transactions on Image Processing 25 (9) (2016) 4444-4457.

[17] D. Lee, K. N. Plataniotis, Toward a no-reference image quality assessment using statistics of perceptual color descriptors, IEEE Transactions on Image Processing 25 (8) (2016) 3875-3889.

[18] K. Gu, D. Tao, J.-F. Qiao, W. Lin, Learning a no-reference quality assessment model of enhanced images with big data, IEEE Transactions on Neural Networks and Learning Systems.

[19] H. R. Sheikh, M. F. Sabir, A. C. Bovik, A statistical evaluation of recent full reference image quality assessment algorithms, IEEE Transactions on Image Processing 15 (11) (2006) 3440-3451.

[20] T. Virtanen, M. Nuutinen, M. Vaahteranoksa, P. Oittinen, J. Häkkinen, CID2013: A database for evaluating no-reference image quality assessment algorithms, IEEE Transactions on Image Processing 24 (1) (2015) 390-402.

${ }_{415}$ [21] M. A. Saad, P. Corriveau, R. Jaladi, Objective consumer device photo quality evaluation, IEEE Signal Processing Letters 22 (10) (2015) 15161520. 
[22] Y. Zhu, G. Zhai, K. Gu, Z. Che, No-reference image quality assessment for photographic images of consumer device, in: 2016 IEEE International Conference on Acoustics, Speech and Signal Processing (ICASSP), IEEE, 2016, pp. 1085-1089.

[23] L. Tang, L. Li, K. Gu, X. Sun, J. Zhang, Blind quality index for camera images with natural scene statistics and patch-based sharpness assessment, Journal of Visual Communication and Image Representation 40 (2016) 335344.

[24] P. J. Huber, Robust statistics, in: International Encyclopedia of Statistical Science, Springer, 2011, pp. 1248-1251.

[25] J.-H. Xue, D. M. Titterington, Median-based image thresholding, Image and Vision Computing 29 (9) (2011) 631-637.

${ }_{430}[26]$ K. N. Chaudhury, A. Singer, Non-local patch regression: Robust image denoising in patch space, in: Acoustics, Speech and Signal Processing (ICASSP), 2013 IEEE International Conference on, IEEE, 2013, pp. 13451349.

[27] K. Arya, P. Gupta, P. K. Kalra, P. Mitra, Image registration using robust m-estimators, Pattern Recognition Letters 28 (15) (2007) 1957-1968.

[28] M. Yang, L. Zhang, J. Yang, D. Zhang, Regularized robust coding for face recognition, IEEE Transactions on Image Processing 22 (5) (2013) 17531766. doi:10.1109/TIP.2012.2235849.

[29] K. Bahrami, A. C. Kot, A fast approach for no-reference image sharpness assessment based on maximum local variation, IEEE Signal Processing Letters 21 (6) (2014) 751-755.

[30] D. L. Ruderman, The statistics of natural images, Network: computation in neural systems 5 (4) (1994) 517-548. 
[31] P. V. Vu, D. M. Chandler, A fast wavelet-based algorithm for global and local image sharpness estimation, IEEE Signal Processing Letters 19 (7) (2012) 423-426.

[32] E. P. Simoncelli, B. A. Olshausen, Natural image statistics and neural representation, Annual review of neuroscience 24 (1) (2001) 1193-1216.

[33] J.-M. Geusebroek, R. Van den Boomgaard, A. W. M. Smeulders, H. Geerts, Color invariance, IEEE Transactions on Pattern Analysis and Machine Intelligence 23 (12) (2001) 1338-1350.

[34] K. Sharifi, A. Leon-Garcia, Estimation of shape parameter for generalized Gaussian distributions in subband decompositions of video, IEEE Transactions on Circuits and Systems for Video Technology 5 (1) (1995) 52-56.

[35] Y. Fang, K. Ma, Z. Wang, W. Lin, Z. Fang, G. Zhai, No-reference quality assessment of contrast-distorted images based on natural scene statistics, IEEE Signal Processing Letters 22 (7) (2015) 838-842.

[36] K. Ghosh, S. Sarkar, K. Bhaumik, Understanding image structure from a new multi-scale representation of higher order derivative filters, Image and Vision Computing 25 (8) (2007) 1228-1238.

[37] Q. Li, W. Lin, Y. Fang, No-reference image quality assessment based on high order derivatives, in: Multimedia and Expo (ICME), 2016 IEEE International Conference on, IEEE, 2016, pp. 1-6.

[38] P. Hall, D. Titterington, J.-H. Xue, Median-based classifiers for highdimensional data, Journal of the American Statistical Association 104 (488) (2009) 1597-1608.

[39] U. Engelke, H. Kaprykowsky, H.-J. Zepernick, P. Ndjiki-Nya, Visual attention in quality assessment, IEEE Signal Processing Magazine 28 (6) (2011) $50-59$. 
[40] L. Zhang, Z. Gu, H. Li, SDSP: A novel saliency detection method by combining simple priors, in: 2013 IEEE International Conference on Image Processing, IEEE, 2013, pp. 171-175.

[41] C. T. Vu, D. M. Chandler, S3: a spectral and spatial sharpness measure, in: Advances in Multimedia, 2009. MMEDIA'09. First International Conference on, IEEE, 2009, pp. 37-43.

[42] N. Ponomarenko, L. Jin, O. Ieremeiev, V. Lukin, K. Egiazarian, J. Astola, B. Vozel, K. Chehdi, M. Carli, F. Battisti, et al., Image database TID2013: Peculiarities, results and perspectives, Signal Processing: Image Communication 30 (2015) 57-77.

[43] L. Ma, W. Lin, C. Deng, K. N. Ngan, Image retargeting quality assessment: A study of subjective scores and objective metrics, IEEE Journal of Selected Topics in Signal Processing 6 (6) (2012) 626-639.

[44] L. Li, W. Xia, W. Lin, Y. Fang, S. Wang, No-reference and robust image sharpness evaluation based on multiscale spatial and spectral features, IEEE Transactions on Multimedia 19 (5) (2017) 1030-1040.

[45] L. Tang, L. Li, K. Sun, Z. Xia, K. Gu, J. Qian, An efficient and effective blind camera image quality metric via modeling quaternion wavelet coefficients, Journal of Visual Communication and Image Representation 49 (2017) 204-212.

[46] L. Tang, L. Li, K. Gu, J. Qian, J. Zhang, No-reference quality assessment of camera-captured distortion images, in: Pacific Rim Conference on Multimedia, Springer, 2016, pp. 590-599.

[47] E. Kee, H. Farid, A perceptual metric for photo retouching, proceedings of the national academy of sciences 108 (50) (2011) 19907-19912.

495

[48] D. Temel, M. Prabhushankar, G. AlRegib, Unique: Unsupervised image quality estimation, IEEE Signal Processing Letters 23 (10) (2016) 14141418 . 


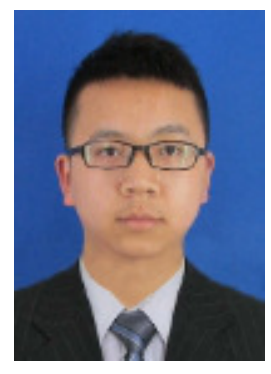

Zhengda Zeng received the B.S. degree in Electronic Information Engineering from Huazhong University of Science and Technology in 2015. He is currently pursuing the M.S. degree with the Department of Electronic Engineering, Tsinghua University. His research interests include image quality assessment and image understanding.

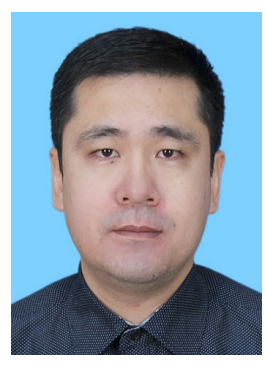

Wenming Yang received his Ph.D. degree in electronic engineering from Zhejiang University in 2006. He is an associate professor in the Department of Electronic Engineering, Graduate School at Shenzhen, Tsinghua University. His research interests include image processing, pattern recognition, computer vision, biometrics, video surveillance and image super-resolution.

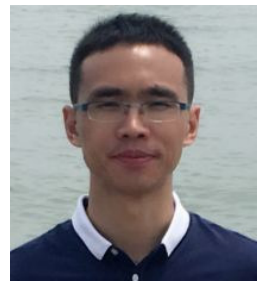

Wen Sun received the B.Eng. degree from the Department of Information Science and Engineering, Southeast University in 2013. He is currently working towards the Ph.D. degree in electronic engineering at Tsinghua University. His research interests include image quality assessment and image understanding.

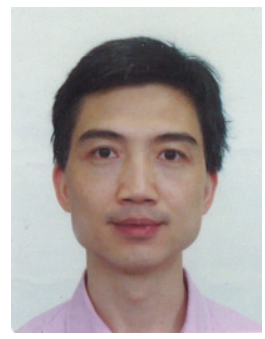

Jing-Hao Xue received the Dr.Eng. degree in signal and information processing from Tsinghua University in 1998 and the Ph.D. degree in statistics from the University of Glasgow in 2008. He is a senior lecturer in the Department of Statistical Science, University College London. His research interests include statistical classification, high-dimensional data analysis, pattern recognition and image analysis. 


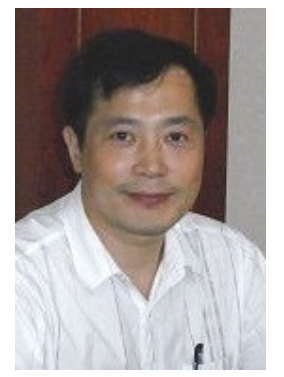

Qingmin Liao received his Ph.D. degree in signal processing and telecommunications from the University of Rennes 1, France, in 1994. He is a Professor in the Department of Electronic Engineering of Tsinghua University, in 2002. Since 2010, he has been the Director of the Division of Information Science and Technology in the Graduate School at Shenzhen,

Tsinghua University. His research interests include image/video processing, transmission, analysis, biometrics and their applications. 